\title{
Evidence and potential risk factors of tuberculosis among captive Asian elephants and wildlife staff in Peninsular Malaysia
}

\begin{abstract}
Elephant tuberculosis (TB) caused by Mycobacterium tuberculosis is an important reemerging zoonosis with considerable conservation and public health risk. We conducted prospective cohort and cross-sectional studies in elephants and wildlife staff respectively in order to identify potential risk factors associated with TB in captive Asian elephants and their handlers in Peninsular Malaysia. Sixty elephants in six different facilities were screened for TB longitudinally using the ElephantTB STAT-PAK and DPP VetTB assays from February 2012 to May 2014, and 149 wildlife staff were examined for tuberculosis infection using the QuantiFERON-TB Gold In-tube (QFT) assay from January to April, 2012. Information on potential risk factors associated with infection in both elephants and staff were collected using questionnaires and facility records. The overall seroprevalence of TB amongst the elephants was $23.3 \%$ (95\% CI: $13.8 \mathrm{i} 36.3$ ) and the risk of seroconversion was significantly higher among elephants with assigned mahouts $[\mathrm{p}=0.022$, OR $=4.9$ (95\% CI: 1.3ї 18.2)]. The percentage of QFT responders among wildlife staff was 24.8\% (95\% CI: 18.3ï 32.7) and the risk of infection was observed to be significantly associated with being a zoo employee [p $=0.018, \mathrm{OR}=2.7(95 \% \mathrm{CI}: 1.2 \mathrm{i}$ 6.3) $]$ or elephant handler $[\mathrm{p}=0.035, \mathrm{OR}=4.1(95 \% \mathrm{CI}$ : 1.1ї 15.5)]. These findings revealed a potential risk of TB infection in captive elephants and handlers in Malaysia, and emphasize the need for TB screening of newly acquired elephants, isolating sero-positive elephants and performing further diagnostic tests to determine their infection status, and screening elephant handlers for TB, pre- and post-employment.
\end{abstract}

Keyword: Captive Asian elephants; Peninsular Malaysia; Risk factors; tuberculosis; Wildlife staff 